\title{
Light emitting diode microscope illumination for green fluorescent protein or fluorescein isothiocyanate epifluorescence
}

\author{
Gottfried Martin, Hansjürgen T. Agostini, and Lutz L. Hansen \\ Universitätsaugenklinik Freiburg, Freiburg, Germany
}

BioTechniques 38:204-206 (February 2005)

Up to now, a microscope is operated with a tungsten halogen lamp for bright field or phase contrast illumination and a mercury arc lamp as a light source for fluorescence. The mercury arc lamp offers bright light sufficient for fluorescence investigation. But there are some shortcomings: the lifetime of the lamp is limited to about $200 \mathrm{~h}$. After this time, the risk of explosion is greatly increased, handling needs a lot of care, it produces a lot of heat, it is very expensive, and it produces harmful UV light even if it is not needed. In our cell culture experiments, we are using an earlier model of an inverted microscope for which fluorescence equipment is not available. Therefore, we looked for an alternative light source. A blue flashlight was used to screen for transgenic green fluorescent protein (GFP) mice in vivo (1). There are some reports in the internet where people used blue light emitting diodes (LEDs) with light intensity below 10 cd with only minor results (http://www. viewsfromscience.com/documents/ webpages/led_fluorescence.html) or as fluorescence illumination for stereomicroscopy (http://130.15.90.245/gfp stereoscope.htm). A portable fluorescence microscope for field use is being developed (http://www.fraen.com). Here we show that the light intensity of new generation LEDs is sufficient for fluorescence investigations and that the quality of the images is comparable to mercury arc lamp illumination.

Two models were used to compare the efficiency of the new light source: $(i)$ GFP-transfected retinal pigment epithelial (RPE) cells and (ii) murine retinal wholemounts after fluorescein isothiocyanate (FITC)-dextran perfusion. RPE cells were cultured as described in (2). They were transfected with a plasmid (pEGFP-
N3; Stratagene, Amsterdam, The Netherlands) conferring expression of GFP. pEGFP-N3 was transfected by effectene (Qiagen, Hilden, Germany) according to the recommendations of the manufacturer. One hundred sixty nanograms pEGFP-N3, $1.3 \mu \mathrm{L}$ enhancer, and $0.8 \mu \mathrm{L}$ effectene were used to transfect 20,000 cells in a well of a 24-well plate passaged the day before. Fluorescein-perfused mouse retina was prepared as described in (3) in a model of oxygen-induced proliferative retinopathy. All animal procedures adhered to the animal care guidelines by the Institute for Laboratory Animal Research (Guide for the Care and Use of Laboratory Animals).

The LED light source was tested both in a normal microscope (BH-2; Olympus, Hamburg, Germany) and an inverted microscope (CK2; Olympus). The standard light source of the $\mathrm{BH}-2$ is the $100 \mathrm{~W}$ highpressure mercury arc lamp of the reflected light fluorescence attachment (BH2RFC). This mercury arc lamp was replaced by a Luxeon Star blue LED [LXHL-MB1C; Conrad, Weiden, Germany; a local electronics supplier) as shown in Figure 1, $\mathrm{A}$ and $\mathrm{B}$. A magnet was mounted on the concave mirror of the lamp housing. The cooling iron of the LED was attached to the magnet so that the
LED could be adjusted perpendicular to the optical axis. Proper adjustment resulted in an intense and homogenous illumination that was easily controlled by putting a sheet of white paper on the field iris as a screen. The LED has a narrow emission spectrum with a maximum at $470 \mathrm{~nm}$ and a half-width of $25 \mathrm{~nm}$ (see data sheet at http://www. luxeon.com/pdfs/protected/DS23. PDF). It was operated with a LED power supply (1 A, 3.5-8 V DC output). There are also LED power supplies with outputs dimmable by a potentiometer. Dimming the light does not influence its spectrum.

The CK2 had no possibility to attach an illumination unit for fluorescence microscopy. A suitable alternative, however, was the filter place below the nosepiece (Figure 1C). The filter cube from the BH2-RFC (IB, exciter filter BP495, dichroitic mirror DM505, barrier filter O515IF; Olympus) with the attached LED (Luxeon Star/O; LXHL-NB98) was mounted there (Figure 1, C and D). This LED is the same as above, but with an optics resulting in a narrow light beam of $15^{\circ}$. The homogenity of the light field was improved by adding a diverging lens [ $f$

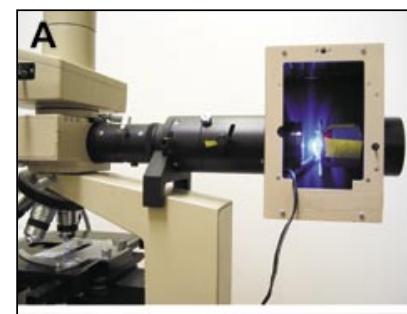

C

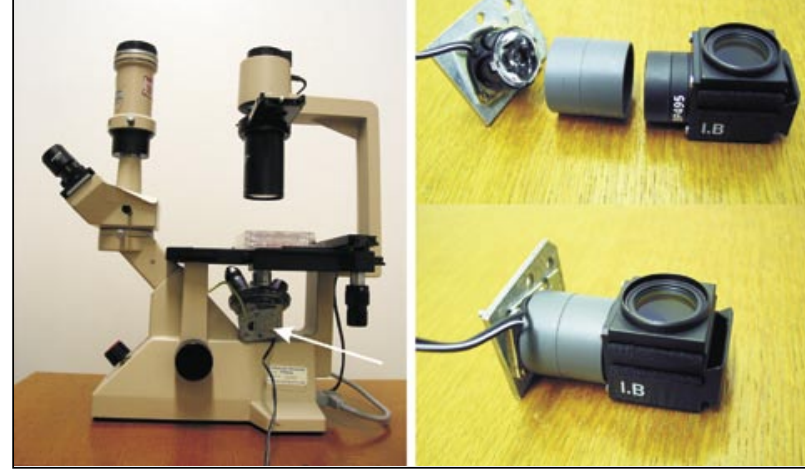

Figure 1. Microscope setup. (A) Normal microscope (BH-2) with light emitting diode (LED) mounted in the lamp housing. (B) LED setup in BH-2. a, collector lens of BH2-RFC; b, LED; c, cooling iron; d, magnet; $\mathrm{e}$, fitting mounted on concave mirror of the lamp housing. (C) Inverted microscope (CK2) with LED (arrow). (D) LED assembly for CK2 with fitting and filter cube. 

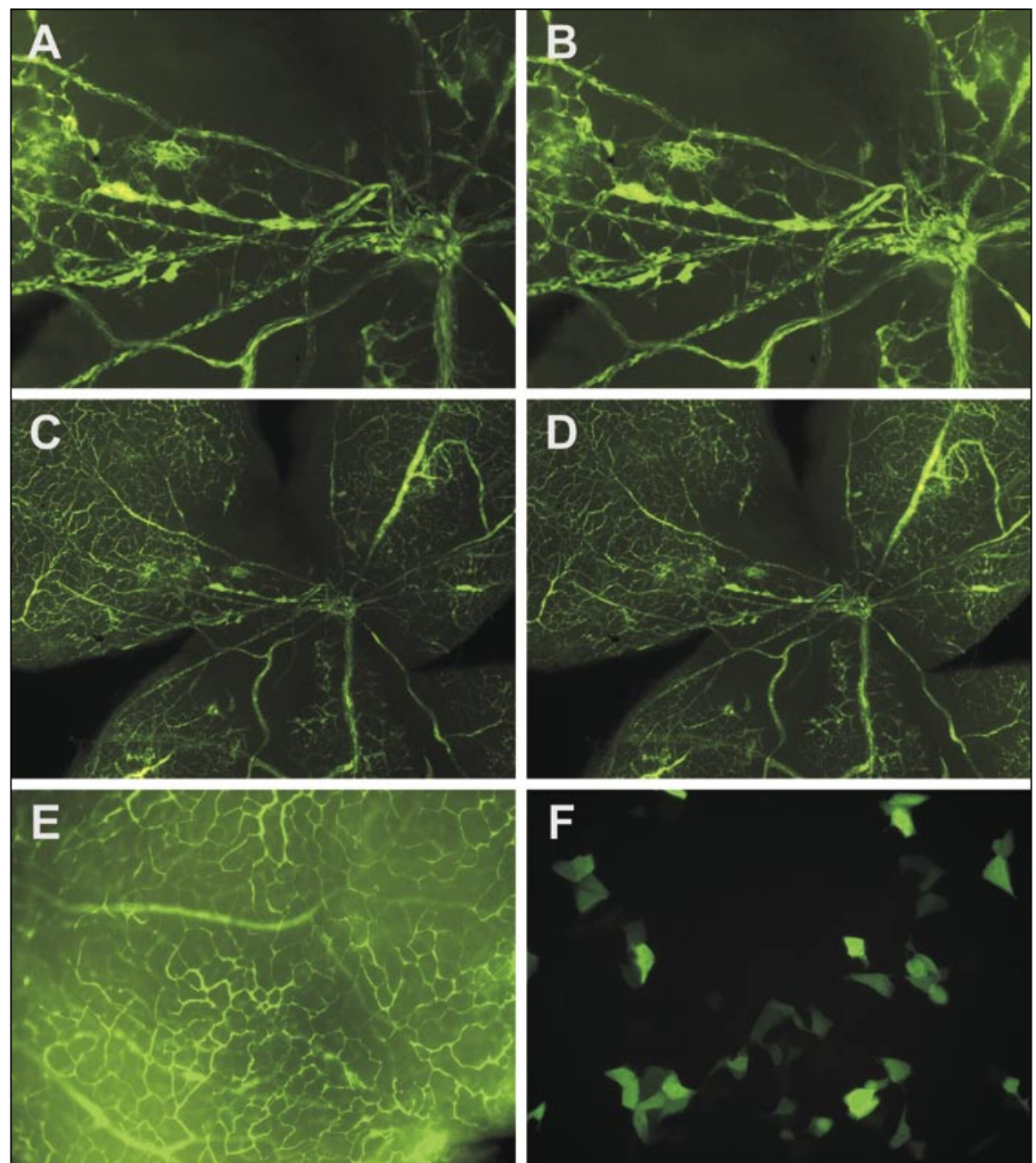

Figure 2. Fluorescent pictures. (A) Retina of mouse perfused with fluorescein isothiocyanate (FITC)dextran with BK-2 and mercury arc lamp. Exposure time $500 \mathrm{~ms}$. 10x objective. (B) Retina of mouse perfused with FITC-dextran with BH-2 and light emitting diode (LED). Exposure time $500 \mathrm{~ms}$. 10x objective. (C) Retina of mouse perfused with FITC-dextran with BH-2 and mercury arc lamp. Exposure time 2 s. $4 \times$ objective. (D) Retina of mouse perfused with FITC-dextran with BH-2 and LED. Exposure time $2 \mathrm{~s} .4 \times$ objective. None of the pictures have been improved by image manipulation. (E) Retina of mouse perfused with FITC-dextran with CK2 and LED. 10x objective. (F) Transfected ARPE-19 cells showing green fluorescent protein (GFP) expression with CK2 and LED. Exposure time 1 s. 10x objective.

$=-33 \mathrm{~mm},-30$ diopter $(\mathrm{dpt})]$ in front of the LED optics.

The fluorescein-perfused mouse retina gave a bright fluorescence in the vessels and capillaries both with mercury arc lamp and LED illumination with the $\mathrm{BH}-2$. Magnifications in the range from $40 \times$ to $400 \times$ were evaluated. LED illumination gave the same light intensity as the mercury arc lamp illumination (compare Figure 2, A and B, and Figure 2, C and D). Corresponding pictures are identical in regard to brightness, homogenity, and resolution of fine structures.
Measurement of the light intensity with a photodiode at the position of the object gave the same values both for LED and mercury arc lamp. With the inverted microscope, the mouse retina gave bright fluorescence in the vessels and capillaries, too (Figure 2E), with all magnifications available ( $40 \times$ to $200 \times$ ). Transfected RPE cells showed clearly visible GFP fluorescence (Figure $2 \mathrm{~F})$. We therefore conclude that LED illumination is an excellent light source for fluorescence microscopy suitable to replace standard mercury arc lamps in the future.

\section{ACKNOWLEDGMENTS}

We thank R. Buchen, B. Flügel, V. Fritz, H. Graner, and A. Mattes for technical assistance and Dr. H. Helm for help with the light measurement. This project was supported by Otto Männer Holding AG.

\section{COMPETING INTERESTS STATEMENT}

The authors declare no competing interests.

\section{REFERENCES}

1.Tyas, D.A., T. Pratt, T.I. Simpson, J.O. Mason, and D.J. Price. 2003. Identifying GFPtransgenic animals by flashlight. BioTechniques $34: 474-476$

2.Schlunck, G., G. Martin, H.T. Agostini, G. Camatta, and L.L. Hansen. 2002. Cultivation of retinal pigment epithelial cells from human choroidal neovascular membranes in age related macular degeneration. Exp. Eye Res. 74:571-576.

3. Unsöld, A.S., B. Junker, R. Mazitschek, G. Martin, L.L. Hansen, A. Giannis, and H.T. Agostini. 2004. Local injection of receptor tyrosine kinase inhibitor MAE 87 reduces retinal neovascularization in mice. Mol. Vis. 10:468-475.

Received 23 September 2004; accepted 29 October 2004.

Address correspondence to Gottfried Martin, Universitätsaugenklinik Freiburg, Killianstr. 5, 79106 Freiburg, Germany. e-mail:martin@aug.ukl.uni-freiburg.de 